\title{
Fitts' Law is modulated by movement history
}

\author{
Rixin Tang ${ }^{1} \cdot$ Bingyao Shen ${ }^{1} \cdot$ Zhiqin Sang $^{1} \cdot$ Aixia Song ${ }^{1} \cdot$ Melvyn A. Goodale ${ }^{2,3,4}$
}

Published online: 24 August 2017

(C) Psychonomic Society, Inc. 2017

\begin{abstract}
Fitts' Law is one of the most robust and wellstudied principles in psychology. It holds that movement time (MT) for target-directed aiming movements increases as a function of target distance and decreases as a function of target width. The purpose of this study was to determine whether Fitts' Law is affected not only by the demands of the target on the current trial but also by the requirements for performance on the previous trial. Experiments 1 and 2 examined trial-totrial effects of varying target width; Experiment 3 examined trial-to-trial effects of varying target distance. The findings from Experiments 1 and 2 showed that moving a finger or cursor towards a large object on a previous trial shortened the movement time on the current trial, whereas the opposite occurred with a small object. In contrast, target distance on the previous trial had no effect on movement time on the current trial. These findings suggest that performance on trial $\mathrm{n}$ has a clear and predictable effect on trial $\mathrm{n}+1$ (at least for target width) and that Fitts' Law as it is normally expressed does not accurately predict performance when the width of the target varies from trial to trial.
\end{abstract}

Rixin Tang

trxtrx518@nju.edu.cn

Melvyn A. Goodale

mgoodale@uwo.ca

1 Department of Psychology, School of Social and Behavioral Sciences, Nanjing University, Nanjing, Jiangsu, People's Republic of China

2 The Brain and Mind Institute, The University of Western Ontario, London, Ontario, Canada

3 Department of Psychology, The University of Western Ontario, London, Ontario, Canada

4 Graduate Program in Neuroscience, The University of Western Ontario, London, Ontario, Canada
Keywords Fitts' Law $\cdot$ Trial history $\cdot$ Task set $\cdot$ Sensorimotor memory

\section{Introduction}

Aiming movements typically become less accurate the faster we make them. This fundamental speed-accuracy trade-off was quantified by Fitts (1954), who proposed that the time required to hit a target is determined by the width of the target and the distance between the start point and target: MT $=\mathrm{a}+$ $\mathrm{b} \times \mathrm{ID}$, where MT is movement time, ID is the index of difficulty, defined as the $\log _{2} 2 \mathrm{~A} / \mathrm{W}$ (A is movement amplitude, W is target width), and $\mathrm{a}$ and $\mathrm{b}$ are empirical constants. This formula, known as Fitts' Law, has been shown to hold across a wide range of tasks, including movements of the hand, handheld tools (Fitts, 1954), or a mouse-controlled cursor (Radwin, Vanderheiden, \& Lin, 1990). Even though there is massive literature about Fitts' Law, little attention has been paid to the possible effects of trial-to-trial transfer of performance. It is true that one previous study has examined the effect of manipulating ID across sequences of trials (Poletti, SleimenMalkoun, Lemaire, \& Temprado, 2016), but there has been no systematic investigation of the effects of manipulating target size and movement amplitude on transfer of performance from trial $n$ to trial $n+1$. This is a critical issue, because there is a long history of work showing that motor programming is a joint product of current information and past performance (for review, see Tang, Whitwell, \& Goodale, 2015).

One clear example of the effects of trial history on current performance comes from studies of grasping. When people grasp an object, maximum grip aperture (MGA) is typically larger on open-loop trials (no visual feedback) than it is on closed-loop trials (visual feedback), presumably because the visuomotor system has to build in a margin of error when 
vision is not available (Jakobson \& Goodale, 1991; Tang, Whitwell, \& Goodale, 2014). But trial history also plays a role: on a closed-loop trial, MGA will be larger if the previous trial was open loop rather than closed loop, and vice versa. Thus, the difference between the MGA on open- and closedloop trials will be pronounced when the two kinds of trials are blocked but much more similar (homogenized) when openand closed-loop trials are randomized or even alternated.

In the current study, we examined the effect of trial history on Fitts' Law where the size or the distance of the target either remained the same from trial to trial or changed. According to Fitts' Law, moving toward a small target or to a target further away should take longer than moving toward a large target or a closer target. However, if trial history plays a role, then the movement time (MT) required to hit the target on a particular trial should be shorter if a large target or a near target was presented on a previous trial and longer if a small object or far object had been presented. In other words, Fitts' Law may be modulated by trial history. In Experiments 1 and 2, we examined the effects of trial-to-trial variation in target width on movement time. In Experiment 1, we used Fitts' classic reciprocal task in which two targets were presented and participants moved between them using either their index finger or a mouse that moved a cursor on the screen. In Experiment 2, we tested participants in discrete trials and again they acquired the target with their finger or with a cursor. In Experiment 3, we examined the effects of trialto-trial variation in target distance for finger movements performed in discrete trials.

\section{Experiments 1A and 1B}

Participants were required to move back and forth between the midline of two rectangles of the same or different widths presented on a touch screen. In Experiment 1A, they used their right index finger. In Experiment 1B, they used a mouse. We predicted that the Movement Time (MT) would reflect not only the width of the current target but also the width of (and thus the required movement to) the previous target.

\section{Method}

\section{Participants and apparatus}

In both experiments, participants were right-handed and had normal or corrected-to-normal vision. Twenty students from Nanjing University ( 9 females, mean age $=20.6$ years) took part in Experiment 1A (index finger) and 22 (15 females, mean age $=21$ ) in Experiment $1 \mathrm{~B}$ (mouse cursor).

Both experiments were conducted using a 43-in ELO touch screen tilted $30^{\circ}$ backward. Viewing distance was approximately $50 \mathrm{~cm}$. In both experiments, participants were presented with four different displays with large (L) and small (S) rectangular targets placed in vertical positions on the left and right sides of the workspace: two large rectangles, two small rectangles, and a small and large rectangle (in one display the small target was on the left and in the other it was on the right; Fig. 1). The distance from the center of each rectangle to the top edge of screen was $27.5 \mathrm{~cm}$. The size of small (S) target was $21.5 \mathrm{~cm} \times 4.6 \mathrm{~cm}$ and large (L) target, $21.5 \mathrm{~cm} \times 9.3 \mathrm{~cm}$. Two different inter-target distances were used: $37.1 \mathrm{~cm}$ (near) or $74.2 \mathrm{~cm}$ (far) from center to center. [Distance, which never varied within a particular block of trials, was not a variable of interest in Experiments 1 and 2.] The eight different displays were presented twice for a total of 16 blocks of trials. We analyzed four different kinds of movements: from large to large (L:L), from small to small (S:S), from large to small $(\mathrm{L}: \mathrm{S})$, and from small to large $(\mathrm{S}: \mathrm{L})$. Note that for the L:S and S:L movements, the MTs obtained with the two displays (small target on left; small target on right) were combined. We also averaged MTs over the near and far distances. Thus, for each participants, we had four different MTs: L:L, S:S, L:S, and S:L.

\section{Procedure and design}

When each block of trials in Experiment 1A (finger) began, the two rectangles were displayed on the screen and participants were instructed to use their right index finger to tap the midline of each target alternately as quickly and as accurately as possible. Half the participants tapped the left rectangle first. If the participant's finger landed outside the target more than twice in a block of trials, the block of trials was begun again. This was true for all experiments in this study. The 16 blocks were randomized and each block lasted for $15 \mathrm{~s}$. Participants rested for $20 \mathrm{~s}$ between blocks. Movement Time was defined as the time between when the subjects lifted their finger from one target to when they placed the finger on the other. The protocol and procedures for Experiment 1B were identical to those of Experiment $1 \mathrm{~A}$, except that the movements were performed with a mouse. In this and all subsequent experiments, participants were given a brief practice session before testing began.

\section{Results and discussion}

In Experiment 1A, two variables (previous size and current size) for L:L, S:S, L:S, and S:L were analyzed. We conducted 2 (Previous size: $\mathrm{L}$ or $\mathrm{S}) \times 2$ (Current size: $\mathrm{L}$ or $\mathrm{S}$ ) repeatedmeasures ANOVAs for MT. The two-way interaction was not significant, $\mathrm{F}(1,19)=0.07, P=0.932, \eta^{2}<0.001$. The main effect of current size was significant, $\mathrm{F}(1,19)=94.203, P<$ $0.001, \eta^{2}=0.832$ : the MT for tapping the small target was longer than the MT for the larger target, a result that is 


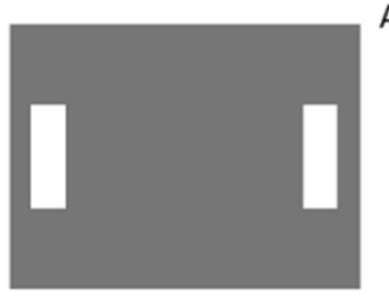

A
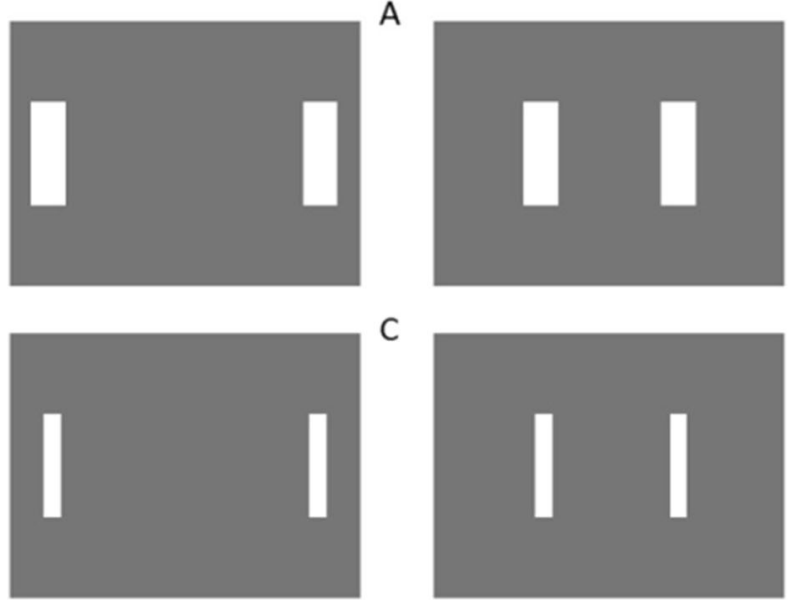

Fig. 1 The target displays used in Experiments 1A and 1B. Note that there were four different kinds of displays, each with two distances between targets. As discussed in the Methods, these displays allowed us

consistent with Fitts' Law. Importantly, the main effect of previous size also was significant, $\mathrm{F}(1,19)=72.926, P<$ $0.001, \eta^{2}=0.793$ : MT was longer if participants had previously tapped a small target, and shorter if they had previously tapped a large target. A paired $t$ test also showed that MT to a large target preceded by a movement to a large target (L:L) was significantly shorter than MT for a large target preceded by a movement to a small target (S:L), $t(19)=6.05, P<0.001$. Similarly, MT to a small target preceded by a movement to a small target (S:S) was significantly longer than MT for a small target preceded by a movement to a large target $(\mathrm{L}: \mathrm{S}), t(19)=$ 5.56, $P<0.001$. These results are illustrated in Fig. 2.

In Experiment 1B, we conducted the same ANOVA as in Experiment $1 \mathrm{~A}$, and the pattern of the results was virtually identical. The main effect of current size $(\mathrm{F}(1,21)=303.976$, $\left.P<0.001, \eta^{2}=0.935\right)$ and previous size $(\mathrm{F}(1,21)=16.172, P$ $\left.=0.001, \eta^{2}=0.435\right)$ were again both significant, and the twoway interaction was not significant, $\mathrm{F}(1,21)=0.724, P=$ $0.404, \eta^{2}=0.033$. As was the case in Experiment 1A, a paired $t$ test showed that MT to a large target that was preceded by a movement to a large target (L:L) was significantly shorter than MT to a large target preceded by a movement to a small target (S:L), $t(21)=2.32, P=0.031$. Similarly, MT to a small target preceded by a movement to a small target (S: S) was significantly longer than MT to a small target preceded by a movement to a large target $(\mathrm{L}: \mathrm{S}), t(21)=3.55, P=0.002$. These results also are illustrated in Fig. 2.

In both Experiments 1A and 1B, we confirmed Fitts' Law (1954): movements to large targets were faster than those to small targets. Importantly, the experiments also showed that the size of the previous target influenced MT to the current target whether the movement was performed with the index finger or the mouse. Movements preceded by movements to a large target were faster than movements
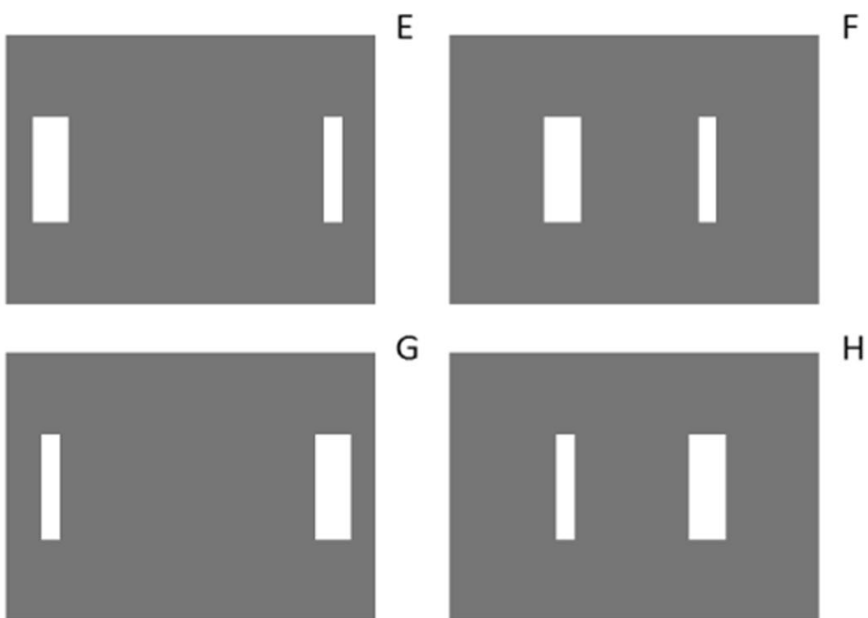

G

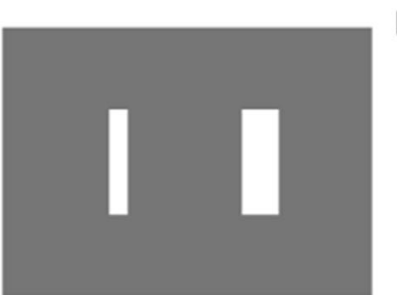

to measure four different kinds of movements: from large to large (L:L), from small to small $(\mathrm{S}: \mathrm{S})$, from large to small $(\mathrm{L}: \mathrm{S})$, and from small to large $(\mathrm{S}: \mathrm{L})$

preceded by movements to a small target, and vice versa. It was still not clear, however, whether or not this occurred only in the traditional reciprocal task, in which time between movements is extremely short. Therefore, we performed a second experiment in which we examined whether the trial-to-trial transfer would also occur in a discretetrial paradigm, in which the time between movements would be longer.

\section{Experiments 2A and 2B}

In Experiment 2A (Finger) and Experiment 2B (Mouse), only one target was presented on each trial and participants were instructed to move their right index finger or the cursor as quickly and accurately as possible to hit the target.

\section{Method}

\section{Participants and apparatus}

The participants who took part in Experiments $1 \mathrm{~A}$ and $1 \mathrm{~B}$ also took part in Experiments 2A and 2B. There was a 2day interval in between. Some participants dropped out, however. In the end, 20 participants were tested in Experiment 2A and 16 in Experiment 2B. The apparatus was the same as in Experiments $1 \mathrm{~A}$ and 1B, but in this case, a white rectangle $(6.7 \mathrm{~cm} \times 4 \mathrm{~cm})$ served as a start box and the target was also a white rectangle (small: $27.8 \mathrm{~cm} \times 3.6 \mathrm{~cm}$ or large: $27.8 \mathrm{~cm} \times 7.2 \mathrm{~cm}$ ). The distance between midline of the start box and the target was either $21.5 \mathrm{~cm}$ or $43 \mathrm{~cm}$. For the layout, see Fig. 3 . 


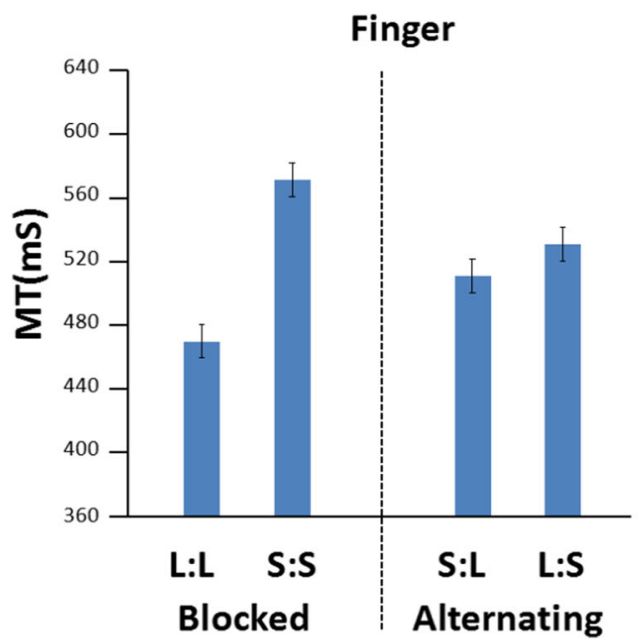

Fig. 2 MT toward current target for the movements made with the index finger and the mouse. In blocked conditions, L:L indicates that both previous target and current target were large. S:S indicates that both previous target and current target were small. In the alternating

\section{Procedure and design}

Before each block of trials began, participants were required to position their finger (Experiment 2A) or the cursor (Experiment 2B) on the start box. As soon as the target appeared on the screen, participants were required to hit the target's midline. The target then disappeared and the participants moved back to the start box. Time interval between trials was $3 \mathrm{~s}$

There were four different kinds of displays: large target near, large target far, small target near, and small target far in Experiments 2A and 2B (Fig. 3). These displays were either blocked or alternated. In the blocked trials, the large target was presented separately at the near distance for 36 trials and at the
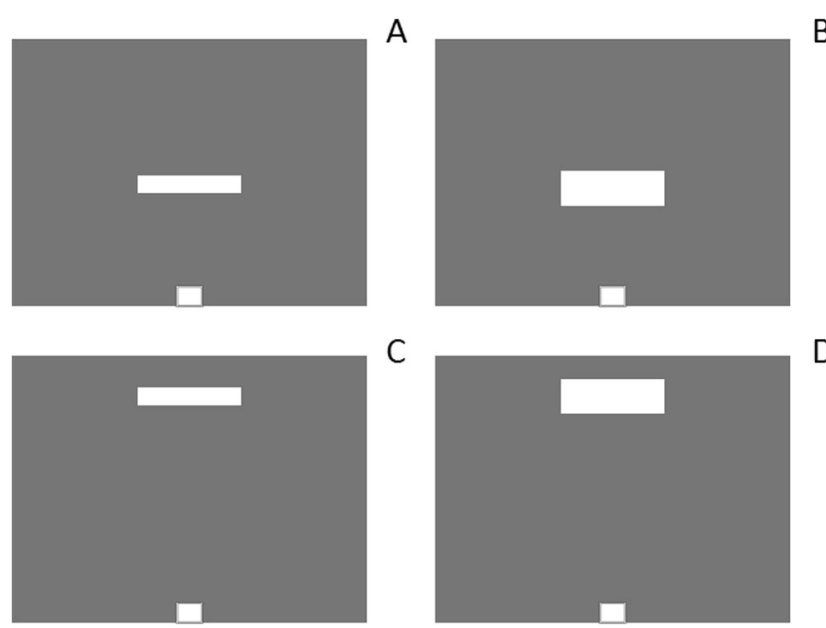

Fig. 3 Stimulus displays used in Experiments 2 and 3. In the blocked trials of Experiments 2 and 3, displays A, B, C, and D were presented in separate blocks of trials. In the alternating trials of Experiment 2, A and B were alternated and $\mathrm{C}$ and $\mathrm{D}$ were alternated. In the alternating trials of Experiment 3, A and C were alternated, and B and D were alternated

\section{Mouse}

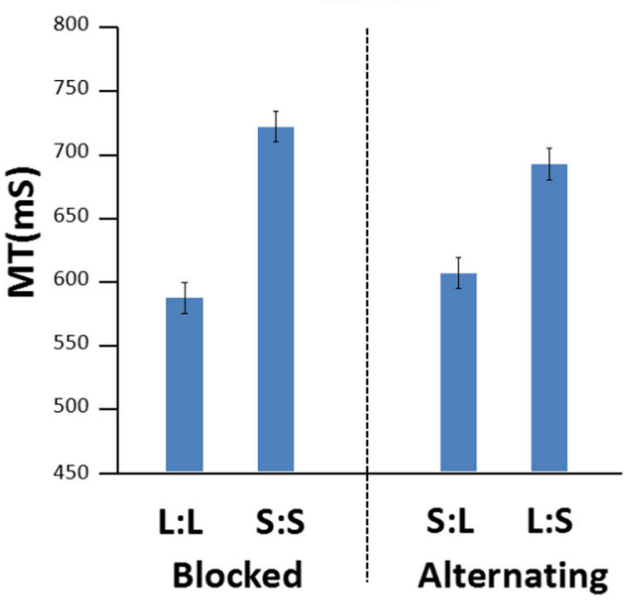

conditions, the size of previous target was different with the size of current target: S:L indicates the size of previous target was small as well as the size of current target was large. L:S indicates the size of previous target was large as well as the size of current target was small

far distance for 36 trials; the same was true for blocked trials with the small target. In the alternating trials, the large and small targets were alternated for 72 trials at the near distance and 72 trials at the far distance. There was a break of $3 \mathrm{~min}$ between trial blocks.

\section{Results and discussion}

The different trial blocks described above were grouped into four different transfer conditions for analysis: large followed by large (L:L), small followed by small (S:S), large followed by small (L:S), small followed by large (S:L). Reaction time did not differ across these transfer conditions in either experiment.

To analyze MT, we conducted a repeated-measures ANOVA (previous size: $\mathrm{L}$ or $\mathrm{S}$ ) $\times 2$ (current size: $\mathrm{L}$ or $\mathrm{S}$ ). The two-way interaction was not significant, $(\mathrm{F}(1,19)=$ $\left.1.189, P=0.289, \eta^{2}=0.059\right)$, but the main effect of current size $\left(\mathrm{F}(1,19)=100.083, P<0.001, \eta^{2}=0.84\right)$ and previous size $\left(\mathrm{F}(1,19)=15.326, P=0.001, \eta^{2}=0.446\right)$ were both significant. Again, paired $t$ tests showed that the MT to the large target preceded by a trial with a large target (L:L) was significantly shorter than MT for a large target preceded by a trial with a small target $(\mathrm{S}: \mathrm{L})(t(19)=2.33, P=0.031)$ and MT to a small target preceded by a trial with a small target $(\mathrm{S}: \mathrm{S})$ was significantly longer than MT to a small target preceded by a trial with a large target $(\mathrm{L}: \mathrm{S}), t(19)=2.95, P=0.008$.

In Experiment 2B (mouse), we performed the identical analysis. The two-way interaction was not significant $\left(\mathrm{F}(1,15)<0.001, P=0.99, \eta^{2}<0.001\right)$, but the main effect of Current size $\left(\mathrm{F}(1,15)=124.441, P<0.001, \eta^{2}=0.892\right)$ and previous size $\left(\mathrm{F}(1,15)=31.238, P<0.001, \eta^{2}=0.676\right)$ were again significant. A paired $t$ test showed that the MT to hit a 
Finger

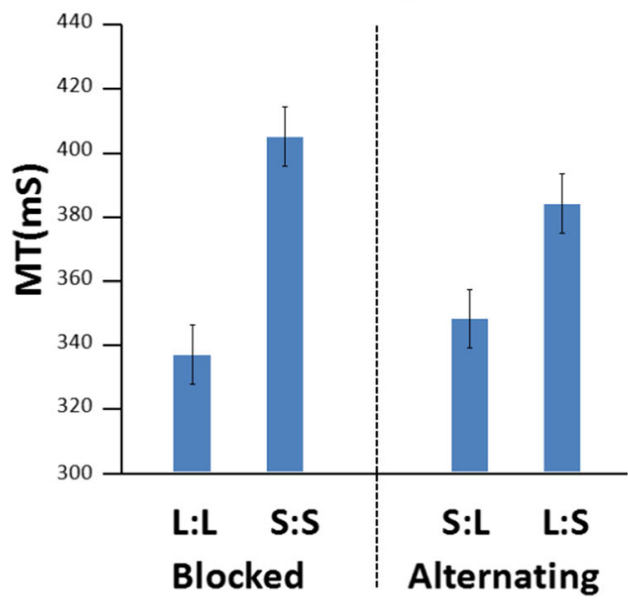

Fig. 4 Movement time to hit targets with the finger and the mouse on current trial. L:L means the target on previous trial was large, and the target on current trial also is large. S:S means the target on previous trial was small, and the target on current trial also is small. S:L means the

large target preceded by a trial with a large target $(\mathrm{L}: \mathrm{L})$ was significantly shorter than MT for a large target preceded by a trial with a small target $(\mathrm{S}: \mathrm{L})(t(15)=3.77, P=0.002)$ and MT to a small target preceded by a trial with a small target $(\mathrm{S}: \mathrm{S})$ was significantly longer than MT to a small target preceded by a trial with a large target (L:S), $t(15)=2.75, P=0.015$ (Fig. 4).

In Experiment 2, we found that time taken to complete an aiming movement was affected by what happened on the previous trial, even in a discrete-trial task, showing that the effect of one trial on the next could bridge several seconds. Moreover, even though participants moved their finger (or the cursor) back to the start box after every movement to the target, this did not affect trial-to-trial transfer.

ID in Fitts' Law also is a function of target distance. In the next experiment, we investigated whether or not MT would be affected by trial history when target distance was blocked or alternated.

\section{Experiment 3}

In Experiment 3, a target of same size was always presented at the same distance in blocked trials or at different distances in alternating trials. Again, participants were instructed to move their right index finger as quickly and accurately as possible to hit the target.

\section{Method}

\section{Participants and apparatus}

Twenty-two participants took part in Experiment 3 (8 males, mean age $=19.8$ years). The apparatus and stimuli

\section{Mouse}

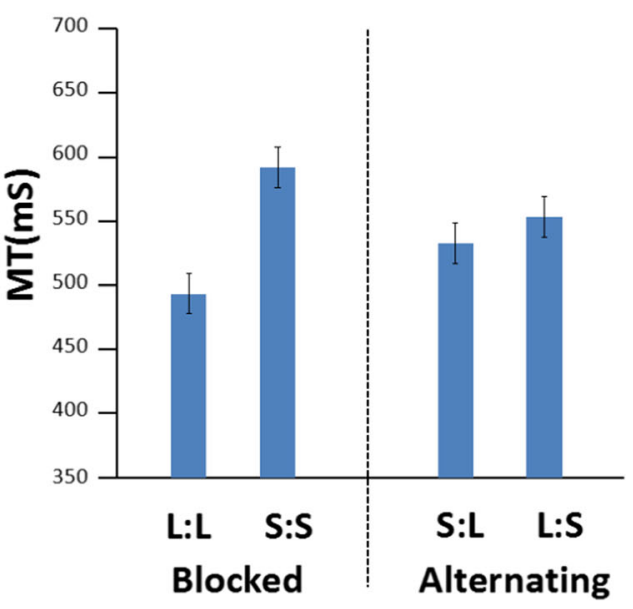

target on previous trial was small, but the target on current trial is large. L:S means the target on previous trial was large, but the target on current trial is small

were identical to those used as in Experiment 2. For the layout, see Fig. 3.

\section{Procedure and design}

The procedure was similar to that used in Experiment 2A except that the size of target was kept constant while the distance was blocked or alternated. As shown in Fig. 3, there were four different kinds of displays: large target near, large target far, small target near, and small target far. In the blocked trials, the large target was presented separately at the near distance for 36 trials and at the far distance for 36 trials; the same was true for blocked trials with the small target. In the alternating trials, far targets were alternated with near targets. This was done separately for small and large targets ( 72 trials in each case). There was a break of 3 min between blocks of trials.

\section{Results and discussion}

The different displays described above were grouped into four different transfer conditions for analysis: far followed by far $(\mathrm{F}: \mathrm{F})$, near followed by near $(\mathrm{N}: \mathrm{N})$, far followed by near $(\mathrm{F}: \mathrm{N})$, near followed by far (N:F). To analyze MT, we conducted a repeated ANOVA, 2 (previous distance: $\mathrm{F}$ or $\mathrm{N}$ ) $\times 2$ (current distance: $\mathrm{F}$ or $\mathrm{N}$ ). The two-way interaction was not significant, $\mathrm{F}(1,21)=0.841, P=0.37, \eta^{2}=0.038$. The main effect of current distance was significant, $\mathrm{F}(1,21)=522.415, P$ $<0.001, \eta^{2}=0.961$. MT was longer for far targets than for near targets. The main effect of previous distance was not significant, $\mathrm{F}(1,21)=0.252, P=0.621, \eta^{2}=0.012$ (Fig. 5).

In Experiment 3, we found no effect whatsoever of trial $n$ on trial $\mathrm{n}+1$ when distance was alternated. The results of all 


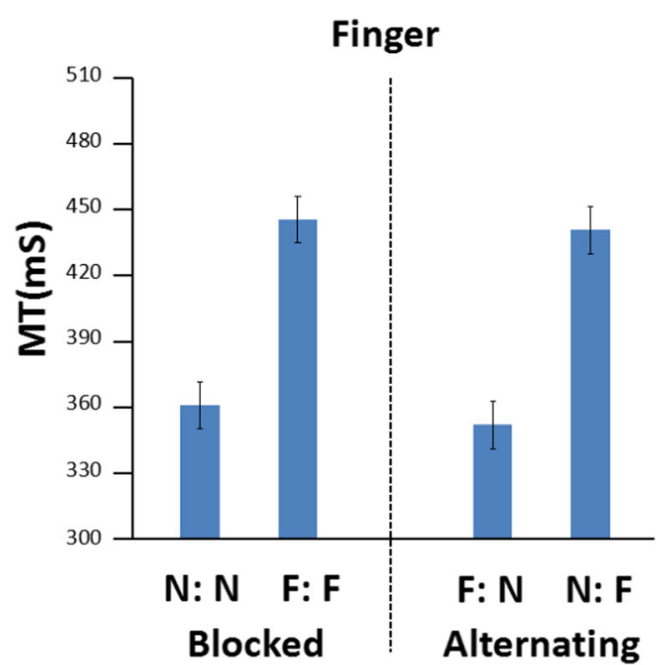

Fig. 5 Movement time to hit targets with the finger and the mouse. N:N means the targets on previous and current trials were presented near to the participant. F:F means the targets on previous and current trials were presented further away from the participant. F:N means the target on previous trial was at a far distance, but the target on current trial was near. $\mathrm{N}: \mathrm{F}$ means the target on previous trial was at near distance, but the target on current trial was far

the experiments show that trial-to-trial variations in target width but not target distance affect MT even though ID is joint product of both variables.

\section{General discussion}

In the first two experiments, the movement was faster when participants reached out to hit the midline of the large target than it was when they reached out to touch the small target-a finding that is consistent with Fitts's Law. This was true whether the participants touched the targets with their finger or moved the cursor to the targets using a mouse. Importantly, however, when participants were presented with the small target on trial $\mathrm{n}$, their movements were slower on trial $\mathrm{n}+1$; conversely, when they were presented with the large target on trial $\mathrm{n}$, their movements were faster on trial $\mathrm{n}+1$. Again, this was true whether the participants touched the targets with their finger or moved the cursor to the targets using a mouse. The clear effect of trial history was observed in both the classic reciprocal tasks (Experiment 1) as well as in the discrete-trial task (Experiment 2).

Importantly, these trial-to-trial transfer effects were not observed in Experiment 3 in which we manipulated target distance from trial to trial. Even though overall, people took longer to reach to more distant targets, an effect that was again consistent with Fitts' Law, the distance of the target on trial $\mathrm{n}$ had no effect on the movement time of trial $\mathrm{n}+1$. It is important to note that in the classic Fitts' Law literature, Index of Difficulty (ID) is a joint function of target width and target distance. Yet, in our study, only trial-to-trial changes in target width was shown to be sensitive to trial history; changes in target distance had no effect. This suggests that a previous experiment (Poletti et al., 2016) that has examined the effects of trial sequences on movement time should not focus only on ID but should examine the effects of target width and target distance separately. Why target width but not target distance should be sensitive to trial history remains a question for future experiments.

Even though there was a clear trial-to-trial effect of target width, it should be noted that in Experiment 1, in which the reciprocal tapping task was used, participants were required to make successive movements in opposite directions. Obviously, different constellations of muscles were used to control these two movements. Tang, Whitwell, \& Goodale (2016) found that motor programming of a grasping movement with the thumb and index finger on a current trial was affected by the performance of a grasping movement using an unfamiliar posture on the previous trial. In some ways, this parallels the findings in Experiment 1 of the current study in that quite different movements were made on successive trials; i.e., the movements were in different directions and alternated between flexion and extension. In Experiment 2, which involved discrete trials, the trial-to-trial transfer occurred for pointing movements made with the finger (or the mouse) in the same direction. It should be noted, however, that this transfer occurred even though a movement back to the start box (in the opposite direction from the aiming movement) was juxtaposed between the two aiming movements. Of course, it could be the case that the movement toward the target somehow influenced the speed with which participants moved back to the start position, and some of this effect transferred to the next target-directed movement. This latter scenario seems unlikely, however, because participants were not instructed to move back to the start position quickly and accurately but simply had to move their finger (or the cursor) back to the start box. Nevertheless, the fact that a movement back towards a start position is typically required in discrete-trial paradigms is something that is not discussed in previous experiments that have looked at the effects of trial history on movement and movement strategies (Song \& Nakayama, 2008; Tang et al., 2015).

Earlier work in our laboratory investigating the effects of trial history on grip aperture in the context of visual feedback strongly suggests that trial-to-trial transfer reflects sensorimotor memory of the last target-directed action, not changes in task set (Tang et al., 2015). It is possible that in the current study, the observed transfer from one movement or trial to the next in Experiment 1 and Experiment 2 respectively also could be due to transfer of some sort of sensorimotor memory. It should be 
acknowledged, however, that participants might employ a different strategy when programming a movement to a large versus a small target, and therefore when confronted with an alternating condition might show transfer of a taskset for a large target when they are later confronted with a small target, and vice versa. On the face of it, this seems unlikely because the same constraints would be operating throughout the task, and all that would be required is plugging information about the size and distance of the target into the motor program. But whatever the mechanism of trial-to-trial transfer might be, it is clear that a robust effect is present and that it can be observed for movements in both the typical reciprocal task as well as a discrete trial version.

In brief, previous research exploring Fitts' Law has focused almost entirely on the movements made on the current trial. Our study makes it clear that performance on trial $\mathrm{n}$ has a clear and predictable effect on trial $\mathrm{n}+1$, at least for target width. Fitts' Law as it is normally expressed does not accurately predict performance when the width of the target varies from trial to trial. To completely capture performance, an additional parameter or some adjustment of the constants needs to be incorporated into the equation to reflect the possible difference in ID (as affected by target width) between trial $\mathrm{n}$ and trial $\mathrm{n}+1$.

Acknowledgements This study was supported by grants from National Natural Science Foundation of China (Grant \#31571131) to R.T. and NSERC (Grant \# 6313) to M.A.G.

\section{References}

Fitts, P. M. (1954). The information capacity of the human motor system in controlling the amplitude of movement. Journal of Experimental Psychology, 47(6), 381-391.

Jakobson, L. S., \& Goodale, M. A. (1991). Factors affecting higher-order movement planning: a kinematic analysis of human prehension. Experimental Brain Research, 86(1), 199-208.

Poletti, C., Sleimen-Malkoun, R., Lemaire, P., \& Temprado, J.-J. (2016) Sensori-motor strategic variations and sequential effects in young and older adults performing a Fitts' task. Acta Psychologica, 163, 19.

Radwin, R. G., Vanderheiden, G. C., \& Lin, M.-L. (1990). A method for evaluating head-controlled computer input devices using Fitts' Law. Human factors, 32(4), 423-438.

Song, J.-H., \& Nakayama, K. (2008). Target selection in visual search as revealed by movement trajectories. Journal of Vision, 48(7), 853861.

Tang, R., Whitwell, R. L., \& Goodale, M. A. (2014). Explicit knowledge about the availability of visual feedback affects grasping with the left but not the right hand. Experimental Brain Research, 232(1), 293302.

Tang, R., Whitwell, R.L., \& Goodale, M.A. (2015). The influence of visual feedback from the recent past on the programming of grip aperture is grasp-specific, shared between hands, and mediated by sensorimotor memory not task set. Cognition, 138, 49-63.

Tang, R., Whitwell, R. L., \& Goodale, M. A. (2016). Unusual hand postures but not familiar tools show motor equivalence with precision grasping. Cognition, 151, 28-36. 